Trauma Surgery \& Acute Care Open

\title{
Retroperitoneal perforation of the descending colon diverticulitis presenting with systemic emphysema
}

\author{
Kaori Kono, ${ }^{1}$ Kaori Ito (1) ,' Yuko Sasajima, ${ }^{2}$ Yasufumi Miyake, ${ }^{1}$ Tetsuya Sakamoto ${ }^{1}$
}

'Division of Acute Care Surgery, Department of Emergency Medicine, Teikyo University School of Medicine, Tokyo, Japan

2Department of Pathology, Teikyo University School of Medicine, Tokyo, Japan

Correspondence to Dr Kaori Ito; kaoriito1@gmail. com

\section{CASE PRESENTATION}

A 75-year-old man was admitted to the cardiac care unit because of congestive heart failure (CHF) secondary to non-ST elevated myocardial infarction (NSTEMI). His medical history included CHF, hypertension, diabetes, bronchial asthma, and hyperuricemia. He denied any past surgical interventions. At admission, the patient was intubated and underwent percutaneous coronary angioplasty (PCA). His hospital course was complicated with refractory respiratory failure due to pulmonary edema, pneumonia, and bronchial asthma. He was intubated three times in 18 days. Steroids used to treat his asthma included intravenous methylprednisolone $40 \mathrm{mg}$ for 3 days, which was switched to oral prednisone $30 \mathrm{mg}$, tapering to $5 \mathrm{mg}$ over 12 days. Ceftriaxone was administered on hospital days 4-11 to treat pneumonia.

$\mathrm{He}$ was finally extubated on hospital day 18, at which time routine chest radiography showed pneumomediastinum, subcutaneous emphysema, and subdiaphragmatic free air (figure 1). He was afebrile and his vital signs were normal except for mild tachycardia (heart rate $105 \mathrm{bpm}$ ). He presented with abdominal bloating but denied abdominal pain. He exhibited crepitation all over his body. His abdomen was distended and soft. His white cell count was $1.21 \times 10 \wedge 9 / \mathrm{L}$, blood urea nitrogen 89.6 $\mathrm{mg} / \mathrm{dL}$, and creatinine $4.35 \mathrm{mg} / \mathrm{dL}$. Non-contrast CT showed extensive subcutaneous emphysema over the chest and abdominal wall, pneumomediastinum without pneumothorax, massive pneumoperitoneum, and pneumoretroperitoneum. Diffuse mesenteric emphysema was observed without ascites (figure 2). The acute care surgery service was consulted.

\section{WHAT WOULD YOU DO?}

A. Start broad-spectrum antibiotics and observe

B. Bronchoscopy, upper and lower gastrointestinal studies

C. Diagnostic laparoscopy

D. Exploratory laparotomy

\section{WHAT WE DID AND WHY}

(c) Author(s) (or their employer(s)) 2020. Re-use permitted under CC BY-NC. No commercial re-use. See rights and permissions. Published by BMJ.

To cite: Kono $\mathrm{K}$, Ito $\mathrm{K}$, Sasajima Y, et al. Trauma Surg Acute Care Open 2020:5:e000606.
Differential diagnoses included the barotrauma due to traumatic intubation/extubation or the increased airway pressure secondary to asthma. Gastrointestinal tract perforation was also suspected. An upper gastrointestinal study showed no contrast leakage from the esophagus through the duodenum. The patient refused a lower gastrointestinal contrast study. At this point, we thought that abdominal exploration to examine the entire bowel was necessary. Diagnostic laparoscopy was therefore discussed, but we decided that the increasing intraabdominal pressure the patient was experiencing from the insufflation made it unsafe because he had undergone PCA for NSTEMI 2 weeks previously. He therefore underwent exploratory laparotomy. On entering the peritoneal cavity, a gush of air was evacuated. There was no free fluid in the peritoneum. Without staining, diffuse emphysema was observed in the small and large intestinal mesenteries and retroperitoneum along with mild emphysema and edema from the descending colon to the sigmoid colon. Diverticula were not observed on the surface of the intestine. Examination of the intestine, from stomach through rectum, showed no perforation or discoloration. At this point, the descending colon was not mobilized from the retroperitoneum because the pneumoretroperitoneum extended over the small and large intestinal mesenteries, and we could not determine the specific area requiring exploration. The abdomen was then closed.

The patient was transferred to the surgical intensive care unit. He was extubated on postoperative day (POD) 1 and transferred to the ward on POD 2. On POD 9, his fever spiked and he presented with left lower quadrant pain, which progressed to septic shock. Blood, sputum, and urine specimens were sent to the laboratory for culture. CT of the chest and abdomen with intravenous and oral contrast revealed fat stranding and extraluminal air with a small amount of free fluid around the descending colon. He was returned to the operating room for re-exploration, during which a small abscess cavity was identified in the mesentery of the descending colon. Hartmann's procedure was performed. The resected specimen revealed a tiny diverticulum penetrating the mesentery and forming an abscess. Pathological analysis results were compatible with perforated diverticulitis (figure 3). His postoperative course was complicated with the need for prolonged mechanical ventilation including tracheostomy. On POD 68/77, he was discharged to a rehabilitation hospital.

\section{DISCUSSION}

We describe a case of retroperitoneal perforation of the descending colon and diverticulitis presenting with systemic emphysema. Diagnosing retroperitoneal penetration of the descending colon with diverticulitis, causing systemic emphysema, was difficult in this patient, who had a complex medical history and vague abdominal symptoms. Because steroid usage and asthma are known risk factors 


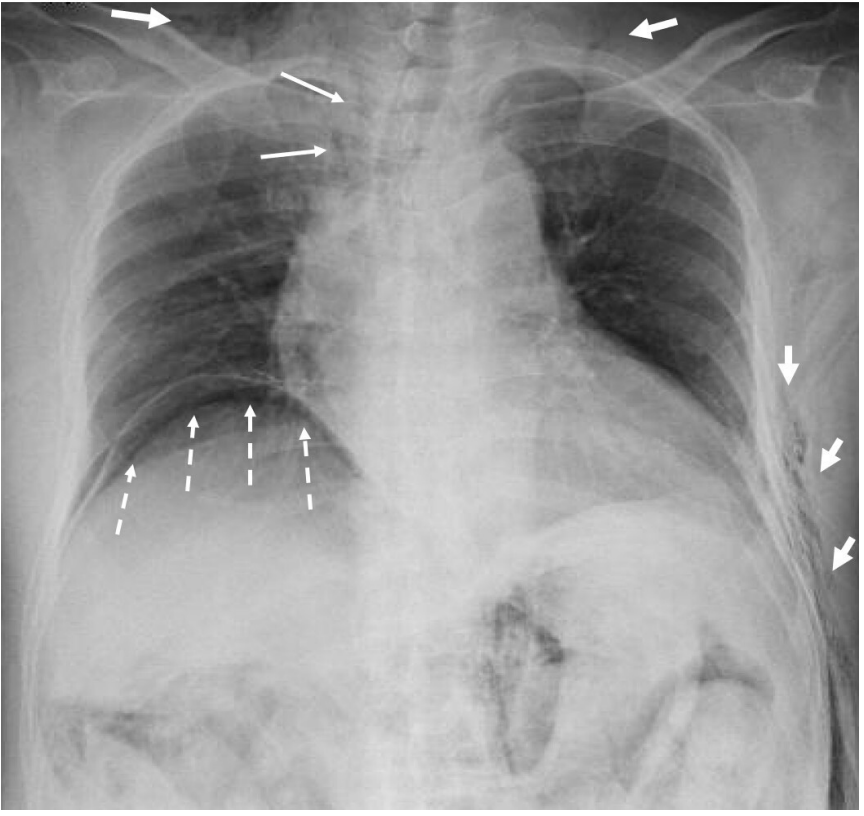

Figure 1 Chest radiograph shows pneumomediastinum (thin arrows), subcutaneous emphysema (thick arrows), and subdiaphragmatic free air (dashed arrows).

for pneumomediastinum, an airway leak was initially suspected. The perforated diverticulum was missed at the initial laparotomy because it was buried in the mesentery and not visible from the outside. In retrospect, we should have mobilized the descending colon from the retroperitoneum or performed intraoperative colonoscopy during the index operation. However, the patient's poor condition, including recent PCA for NSTEMI, precluded prolonging the operation for extra procedures.

According to the current guidelines, non-operative management is recommended for perforated colonic diverticulitis without purulent or feculent peritonitis (Hinchey stage III or IV). This patient's condition could also be described as progression

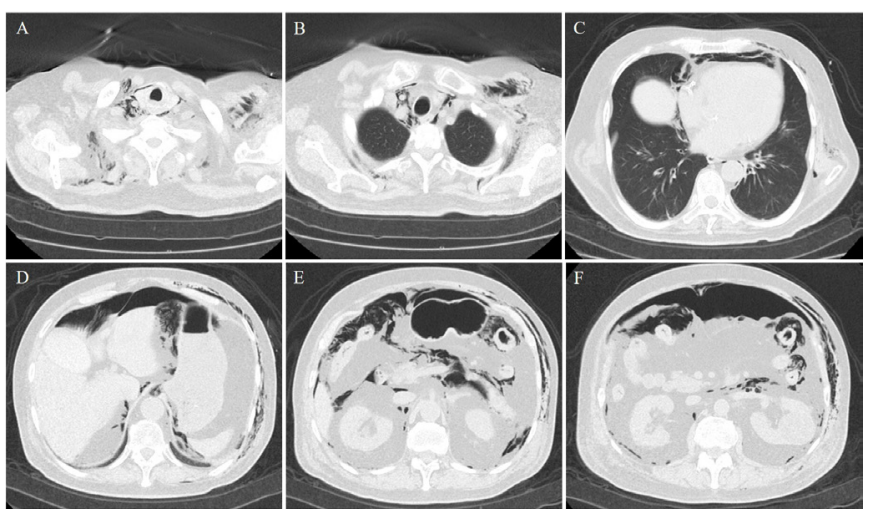

Figure 2 CT of the chest and abdomen reveals subcutaneous emphysema $(A-F)$, pneumomediastinum $(A, B, C)$, pneumoperitoneum $(C, D, E)$, pneumoretroperitoneum $(C, D, E)$, and mesenteric emphysema $(D, E)$.

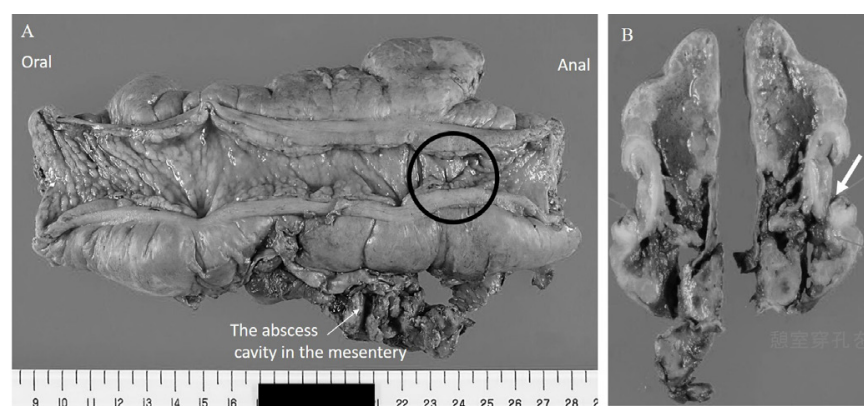

Figure 3 Macroscopically, the resected colon had scattered diverticula. The perforation was not visible in the mucosa (black circle in A). There is a deep insertion that seems to be perforation on the split surface of the specimen (arrow in B). There is a stool influx that may be macroscopically due to the perforation. It is conjectured that there was diverticulitis and associated perforation.

of disease secondary to microperforation with a tiny mesenteric abscess (Hinchey stage I), which typically does not mandate emergency surgery. The delayed diagnosis in this patient placed him at high risk of serious consequences, such as septic shock. It is thus important to recognize that perforated diverticulitis presenting with systemic emphysema is an alarming condition even without purulent or feculent peritonitis.

Systemic emphysema secondary to non-iatrogenic colonic perforation is a rare complication. To date, only 20 such cases have been reported. The site of perforation in all cases, including this one, was the retroperitoneal or mesenteric space. The most common causes of systemic emphysema of colonic origin are diverticulitis, ulcerative colitis, and colorectal cancer, in descending order. Among the 20 reported patients, three died from the condition.

Although systemic emphysema due to retroperitoneal penetration of the descending colonic diverticulum is serious, the abdominal findings may be unclear because it does not cause peritonitis until the free perforation occurs. It is thus important to have a high index of suspicion of retroperitoneal penetration due to colonic diverticulitis when systemic emphysema is observed even in patients without peritoneal signs.

Contributors Conception and design: KK, KI. Acquisition of data: KK. Drafting of the manuscript: KK, KI. Critical revision of the manuscript: YM, TS. Supervision: YM, TS.

Funding The authors have not declared a specific grant for this research from any funding agency in the public, commercial or not-for-profit sectors.

Competing interests None declared.

Patient consent for publication Obtained.

Provenance and peer review Not commissioned; externally peer reviewed.

Open access This is an open access article distributed in accordance with the Creative Commons Attribution Non Commercial (CC BY-NC 4.0) license, which permits others to distribute, remix, adapt, build upon this work non-commercially, and license their derivative works on different terms, provided the original work is properly cited, appropriate credit is given, any changes made indicated, and the use is non-commercial. See: http://creativecommons.org/licenses/by-nc/4.0/.

ORCID iD

Kaori Ito http://orcid.org/0000-0001-5276-2064 